p53

\section{Role model}

Christmas might have come early for researchers studying p53 mechanisms. Papers by Brent Derry and colleagues in Science, and by Schumacher and co-workers in Current Biology report the identification of a 553 homologue in Caenorhabditis elegans, and show that it is involved in DNA-damagestress resistance. Their findings could provide the genetic model that scientists have been waiting for to uncover the pathways through which $\mathrm{p} 53$ functions. induced apoptosis, meiosis and
As previous analyses of the C. elegans genome had not detected a 553 homologue, it was generally assumed that $\mathrm{p} 53$ is a mammalian-specific gene. But this idea was challenged last year with the identification of a Drosophila homologue although the organism-wide role of the gene could not be assesed in these experiments. So, the teams involved in the new studies re-examined the $C$. elegans sequence in more detail and discovered there is, in fact, a similar gene to p53, which Brent Derry and colleagues named cep-1 (C. elegans p53-like 1).

\section{PROTEIN PROCESSING}

\section{A guiding star}

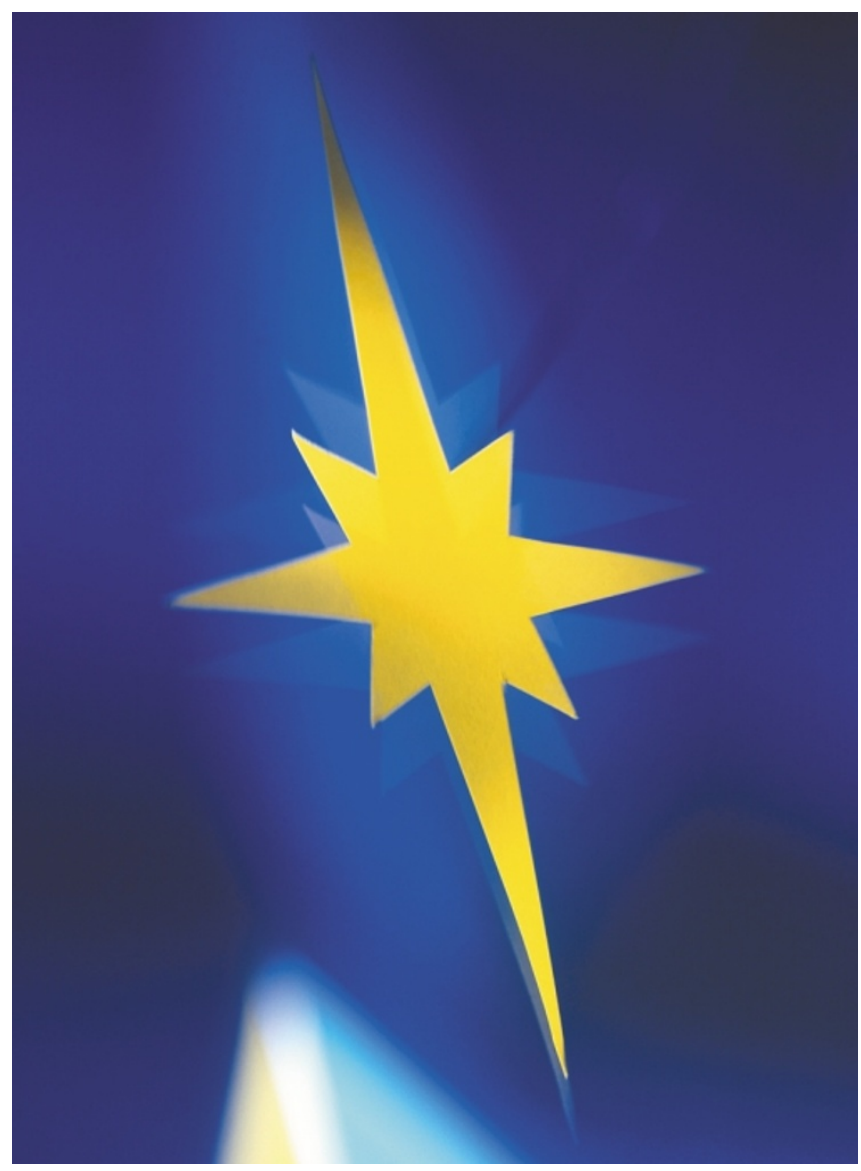

Both teams then set out to identify the role of this homologue. RNA interference studies by both groups showed that the inhibition of cep-1 affected radiation-induced apoptosis in the germ line (and not somatic cell death), but did not affect DNA-damageinduced cell-cycle arrest. Therefore, p53 might be responsible for maintaining the fidelity of germ cells by eliminating compromised cells. Further studies by Brent Derry and colleagues revealed more key phenotypes in cep-1 mutants - they showed meiotic chromosome stability defects, sensitivity to hypoxia, and diminished life span during starvation. Fusion-protein studies by Schumacher and colleagues found that, like its

According to genetic studies, Spitz - the main ligand of the Drosophila melanogaster epidermal growth factor (EGF) receptor must undergo proteolysis to release its extracellular EGF domain. Two transmembrane proteins, Star and Rhomboid-1 (one of a family of seven Rhomboids), have been linked with this process. So far, supporting biological data have been elusive, but back-to-back papers in Cell by Freeman and colleagues at last reveal Star and Rhomboid's function, and in doing so uncover a new mechanism of growth-factor activation.

To begin with, the authors showed that expressing both recombinant Star and Rhomboid1 in Drosophila embryos induced the cleavage of a myc-tagged form of Spitz; and they confirmed that Star and Rhomboid-1 are sufficient for Spitz cleavage using a cellculture model. Immunostaining showed that when the proteins were expressed individually, Spitz localized to the endoplasmic reticulum (ER), Rhomboid-1 to the Golgi, and Star to the ER, Golgi and plasma membrane.

However, coexpression of Star with Spitz caused Spitz to relocate to the Golgi, where it colocalized with Rhomboid-1. So the function human counterpart, CEP-1 acts as a transcription factor. Attempts to find the gene structure of cep- 1 have produced differing results. Based on computer predictions, Brent Derry and colleagues found that the putative complementary DNA sequence predicts a 429 -amino-acid protein that is similar to the human protein in the aminoterminal and DNA-binding regions. However, Schumacher and colleagues, who isolated the cDNA by PCR amplification, showed the putative sequence was 645 amino acids in length, with lower sequence homology to other species, as it only showed similarity around the DNA-binding region. Further work will be needed to confirm these gene structures.

of Star is to chaperone Spitz from the ER to the Golgi, where it is cleaved - and thereby activated — by Rhomboid-1. Once cleaved, the soluble lumenal fragment is secreted from the cell. Regulating Drosophila EGF-receptor activation therefore begins in the signalemitting cell. Further analysis showed that the lumenal domain of Spitz, with some help from its cytoplasmic domain, seems to be necessary for Star-mediated localization. Reciprocally, Star requires its lumenal domain to relocalize Spitz, but so far it's not known whether this interaction blocks a Spitz ER-retention signal, or whether Star somehow actively exports Spitz to the Golgi.

Once Spitz is in the Golgi, it gets cleaved by Rhomboid-1. But which part of Spitz does Rhomboid-1 target? The authors showed the answer was the transmembrane domain. Whereas the mammalian homologue of Spitz, transforming growth factor- $\alpha$, is cleaved by a metalloprotease, Freeman's group propose in the second paper that Rhomboid-1 is itself a serine protease, which defines a new mechanism of growth-factor activation. Unusually for a serine protease, Rhomboid's active site resides within the membrane bilayer, con- 
As genetic methods in C. elegans models have been a crucial factor in the identification of several cellular pathways, the authors of both papers hope their findings will provide new insights into the regulation and function of mammalian p53. Another potential key area of research could spring from Brent Derry and colleagues' isolation of a genomic p53 mutation, which could provide the possibility for screening tools.

Simon Frantz

(2) References and links ORIGINAL RESEARCH PAPERS Brent Derry, W., Putzke, A. P. \& Rothman, J. H. Caenorhabditis elegans p53: role in apoptosis, meiosis and stress resistance. Science 294, 591-595 (2001) | Schumacher, B., Hofmann, K., Boulton, S. \& Gartner, A. The C. elegans homolog of the p53 tumor suppressor is required for DNA damageinduced apoptosis. Curr. Biol. 11, 1722-1727 (2001)

sistent with the finding that Spitz is cleaved within its transmembrane domain - a process known as regulated intracellular proteolysis (RIP). However, this mechanism represents a new type of RIP, as, in contrast to previous examples where factors are released into the cytoplasm, Spitz is released as an extracellular factor.

As is often the case, answering one question leads to a fresh foray. Not least is whether Rhomboids are involved in activating EGF receptors in humans, as the Rhomboid family seems to be evolutionarily conserved from archaea and bacteria to humans. If this turns out to be the case, Rhomboids might become an attractive new target for drugs. As Drosophila contains another six Rhomboid proteins, a next step will be to work out what they do.

Katrin Bussell

69) References and links ORIGINAL RESEARCH PAPERS Lee, J. R. et al. Regulated intracellular ligand transport and proteolysis control EGF signal activation in Drosophila. Cell 107, 161-171 (2001) | Urban, S. Lee, J. R. \& Freeman, M. Drosophila Rhomboid-1 defines a family of putative intramembrane serine proteases. Cell 107, 173-182 (2001)

FURTHER READING Wasserman, $J$. D. et al. A family of Rhomboid-like genes: Drosophila rhomboid-1 and roughoid/rhomboid-3 cooperate to activate EGF receptor signalling. Genes Dev. 14, 1651-1663 (2000)

WEB SITE

Matthew Freeman's laboratory:

http://www2.mrc-Imb.cam ac uk/research/CB/

Freeman_M/Freeman_M.html
CELL CYCLE

\section{Switching on S phase}

The process by which cells divide consists of a cyclical series of phases, and the transition from one to the next is generally controlled by the expression, modification and destruction of various proteins. The switch from G1 phase to $S$ phase in yeast, for instance, requires the breakdown of the Sicl protein. Writing in the 29 November issue of Nature, Mike Tyers, Tony Pawson and colleagues show that this process has a built-in timer mechanism, which involves the accumulation of at least six phosphate groups on Sicl.

Yeast cells are driven into $S$ phase by a complex that consists of a cyclin-dependent kinase (known as Cdc28) and its cyclin partners (Clb5/Clb6). During G1 phase, this complex is inhibited by Sic1, which ensures that cells do not enter $S$ phase prematurely. At the G1/S transition, Sicl is destroyed and $\mathrm{Clb}-\mathrm{Cdc} 28$ is no longer blocked.

A prerequisite for the destruction of Sic1 is that it is phosphorylated (by Cdc28 in complex with another set of cyclins, $C \ln 1 / C \ln 2 / C \ln 3$ ); this enables Sicl to be recognized by the cellular protein-degrading machinery through a protein called Cdc4. It was already known that Sicl is phosphorylated many times before it can be detected and destroyed. But what wasn't clear was why this was the case.

To find out, Tyers and colleagues mutated the nine sites in Sicl that can be phosphorylated by Cln-Cdc28. They then added the sites back, one at a time. They showed that the protein needs at least six of these sites before it can bind to Cdc4 or be degraded in vivo. Next, they identified a consensus amino-acid sequence surrounding a phosphorylation site that can bind to $\mathrm{Cdc} 4$ with high affinity. Cyclin $\mathrm{E}$ has this sequence, but none of the nine phosphorylation sites in Sicl fits the consensus perfectly. Yet there is strength in numbers. Individually, these sites in Sic1 bind only weakly to $\mathrm{Cdc} 4$, but together they make a high-affinity Cdc4-binding site.

Moreover, it is biologically important that Sicl has so many phosphorylation sites, as Tyers and colleagues discovered when they created a Sic1 protein that contained the single, high-affinity Cdc4-binding site from cyclin $\mathrm{E}$. The hybrid protein was destroyed too efficiently, leading to premature entry into $S$ phase and consequent genome instability.

All of which leads to the idea that cells time their entry into $S$ phase to perfection by using a phosphate-dependent 'fuse'. If a single

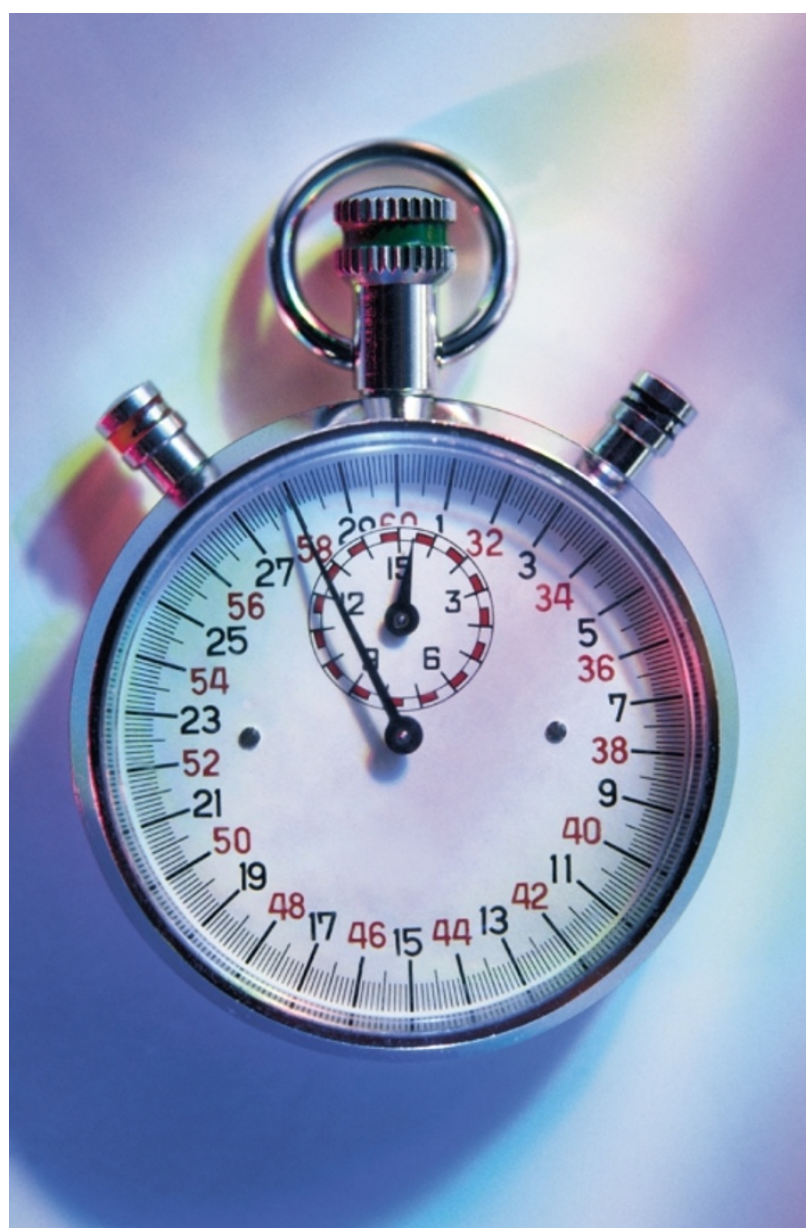

phosphorylation site were sufficient for Sicl to be recognized and destroyed, then even a small amount of Cln-Cdc28 activity would drive cells into $S$ phase, perhaps prematurely. But the fact that at least six sites need to be phosphorylated before Sic1 is destroyed ensures that a threshold level of Cln-Cdc28 activity must be reached. The use of this timer mechanism also means that the transition into $S$ phase is inherently switch-like, in that it depends on the sixth order of the Cln-Cdc28 concentration in a highly nonlinear response.

Amanda Tromans, Associate Editor, Nature

(2) References and links

ORIGINAL RESEARCH PAPER Nash, P. et al. Phosphorylation of many sites of a CDK inhibitor sets a threshold for the onset of $S$ phase. Nature 414, 516-523 (2001)

FURTHER READING Bartek, J. \& Lukas, J. Pathways governing G1/S transition and their response to DNA damage. FEBS Lett. 490, 117-122 (2001) | Rhind, N. \& Russell, P. Checkpoints: it takes more than time to heal some wounds. Curr. Biol. 10, R908-R911 (2000)

\section{WEB SITES}

Mike Tyers' laboratory: http://www.mshrion.ca/tyers

Focus on Cell Division: http://www.nature.com/celldivision 